\title{
A SIMPLE PROOF OF A CONGRUENCE FOR A SERIES INVOLVING THE LITTLE $q$-JACOBI POLYNOMIALS
}

\author{
ATUL DIXIT \\ Dedicated to Professor George E. Andrews on the occasion of his 80th birthday
}

\begin{abstract}
We give a simple and a more explicit proof of a mod 4 congruence for a series involving the little $q$-Jacobi polynomials which arose in a recent study of a certain restricted overpartition function.
\end{abstract}

\section{INTRODUCTION}

In [3, Andrews, Schultz, Yee and the author studied the overpartition function $\bar{p}_{\omega}(n)$, namely, the number of overpartitions of $n$ such that all odd parts are less than twice the smallest part, and in which the smallest part is always overlined. In the same paper, they obtained a representation for the generating function of $\bar{p}_{\omega}(n)$ in terms of a ${ }_{3} \phi_{2}$ basic hypergeometric series and an infinite series involving the little $q$-Jacobi polynomials. The latter are given by [2, Equation (3.1)]

$$
p_{n}(x ; \alpha, \underline{q}):={ }_{2} \phi_{1}\left(\begin{array}{c}
q^{-n}, \\
\alpha \underline{q}^{n+1} \\
\alpha q
\end{array} ; q x\right),
$$

where the basic hypergeometric series ${ }_{r+1} \phi_{r}$ is defined by

$$
{ }_{r+1} \phi_{r}\left(\begin{array}{c}
a_{1}, a_{2}, \ldots, a_{r+1} \\
b_{1}, b_{2}, \ldots, b_{r}
\end{array} ; q, z\right):=\sum_{n=0}^{\infty} \frac{\left(a_{1} ; q\right)_{n}\left(a_{2} ; q\right)_{n} \cdots\left(a_{r+1} ; q\right)_{n}}{(q ; q)_{n}\left(b_{1} ; q\right)_{n} \cdots\left(b_{r} ; q\right)_{n}} z^{n},
$$

and where we use the notation

$$
\begin{aligned}
& (A ; q)_{0}=1 ; \quad(A ; q)_{n}=(1-A)(1-A q) \cdots\left(1-A q^{n-1}\right), \quad n \geq 1, \\
& (A ; q)_{1}=\lim _{n \rightarrow 1}(A ; q)_{n} \quad(|q|<1) .
\end{aligned}
$$

The precise representation for the generating function of $\bar{p}_{\omega}(n)$ obtained in [3] is as follows.

Theorem 1.1. The following identity holds for $|q|<1$ :

$$
\begin{aligned}
& \bar{P}_{\omega}(q):=\sum_{n=1}^{\infty} \bar{p}_{\omega}(n) q^{n}=-\frac{1}{2} \frac{(q ; q)_{\infty}\left(q ; q^{2}\right)_{\infty}}{(-q ; q)_{\infty}\left(-q ; q^{2}\right)_{\infty}}{ }_{3} \phi_{2}\left(\begin{array}{rll}
-1, & i q^{1 / 2}, & -i q^{1 / 2} \\
q^{1 / 2}, & -q^{1 / 2}
\end{array}\right) \\
& +\frac{(-q ; q)_{\infty}}{(q ; q)_{\infty}} \sum_{n=0}^{\infty} \frac{\left(q ; q^{2}\right)_{n}(-q)^{n}}{\left(-q ; q^{2}\right)_{n}\left(1+q^{2 n}\right)} p_{2 n}\left(-1 ; q^{-2 n-1},-1: q\right) .
\end{aligned}
$$

\footnotetext{
${ }^{1} 2010$ Mathematics Subject Classification: Primary, 11P81; Secondary, 05A17

${ }^{2}$ Keywords and phrases: overpartitions, congruence, little $q$-Jacobi polynomials
} 
Later, Bringmann, Jennings-Shaffer and Mahlburg [4, Theorem 1.1] showed that $\bar{P}_{\omega}(q)+$ $\frac{1}{4}-\frac{\eta(4 \tau)}{2 \eta(2 \tau)^{2}}$, where $q=e^{2 \pi i \tau}$ and $\eta(\tau)$ is the Dedekind eta function, can be completed to a function $\hat{P}_{\omega}(\tau)$, which transforms like a weight 1 modular form. They called the function $\bar{P}_{\omega}(q)+\frac{1}{4}-\frac{\eta(4 \tau)}{2 \eta(2 \tau)^{2}}$ a higher depth mock modular form.

While the series involving the little $q$-Jacobi polynomials in Theorem 1.1 itself looks formidable, it was shown in [3, Theorem 1.3] that modulo 4, it is a simple $q$-product. The mod 4 congruence proved in there is given below.

Theorem 1.2. The following congruence holds:

$$
\sum_{n=0}^{\infty} \frac{\left(q ; q^{2}\right)_{n}(-q)^{n}}{\left(-q ; q^{2}\right)_{n}\left(1+q^{2 n}\right)} p_{2 n}\left(-1 ; q^{-2 n-1},-1: q\right) \equiv \frac{1}{2} \frac{\left(q ; q^{2}\right)_{\infty}}{\left(-q ; q^{2}\right)_{\infty}} \quad(\bmod 4) .
$$

The proof of this congruence in [3] is beautiful but somewhat involved. The objective of this short note is to give a very simple proof of it. In fact, we derive it as a trivial corollary of the following result.

Theorem 1.3. For $|q|<1$, we have

$$
\begin{aligned}
& \sum_{n=0}^{\infty} \frac{\left(q ; q^{2}\right)_{n}(-q)^{n}}{\left(-q ; q^{2}\right)_{n}\left(1+q^{2 n}\right)} p_{2 n}\left(-1 ; q^{-2 n-1},-1: q\right) \\
& =\frac{1}{2} \frac{\left(q ; q^{2}\right)_{\infty}}{\left(-q ; q^{2}\right)_{\infty}}+\frac{4 q^{2}}{(1+q)} \sum_{n=0}^{\infty} \frac{\left(q^{3} ; q^{2}\right)_{n}(-q)^{n}}{\left(-q^{3} ; q^{2}\right)_{n}\left(1+q^{2 n+2}\right)} \sum_{j=0}^{n} \frac{(-q ; q)_{2 j} q^{2 j}}{\left(q^{2} ; q\right)_{2 j}}
\end{aligned}
$$

The presence of 4 in front of the series on the right-hand side in the above equation immediately implies that Theorem 1.2 holds.

\section{Proof of Theorem 1.3}

Observe that from (1.1),

$$
\sum_{n=0}^{\infty} \frac{\left(q ; q^{2}\right)_{n}(-q)^{n}}{\left(-q ; q^{2}\right)_{n}\left(1+q^{2 n}\right)} p_{2 n}\left(-1 ; q^{-2 n-1},-1: q\right)=\sum_{n=0}^{\infty} \frac{\left(q ; q^{2}\right)_{n}(-q)^{n}}{\left(-q ; q^{2}\right)_{n}\left(1+q^{2 n}\right)} \sum_{j=0}^{2 n} \frac{(-1 ; q)_{j}}{(q ; q)_{j}}(-q)^{j} \text {. }
$$

However, let us first consider

$$
A(q):=\sum_{n=0}^{\infty} \frac{\left(q ; q^{2}\right)_{n}(-q)^{n}}{\left(-q ; q^{2}\right)_{n}\left(1+q^{2 n}\right)} \sum_{j=0}^{2 n} \frac{(-1 ; q)_{j}}{(q ; q)_{j}} q^{j} .
$$

The only difference in the series on the right-hand side of (2.1) and the series in (2.2) is the presence of $(-1)^{j}$ inside the finite sum in the former.

To simplify $A(q)$, we start with a result of Alladi [1, p. 215, Equation (2.6)]:

$$
\frac{(a b q ; q)_{n}}{(b q ; q)_{n}}=1+b(1-a) \sum_{j=1}^{n} \frac{(a b q ; q)_{j-1} q^{j}}{(b q ; q)_{j}}
$$


Let $a=-1, b=1$ and replace $n$ by $2 n$ so that

$$
\sum_{j=0}^{2 n} \frac{(-1 ; q)_{j} q^{j}}{(q ; q)_{j}}=\frac{(-q ; q)_{2 n}}{(q ; q)_{2 n}} .
$$

Substitute (2.4) in (2.2) to see that

$$
\begin{aligned}
A(q) & =\sum_{n=0}^{\infty} \frac{\left(q ; q^{2}\right)_{n}(-q)^{n}}{\left(-q ; q^{2}\right)_{n}\left(1+q^{2 n}\right)} \frac{(-q ; q)_{2 n}}{(q ; q)_{2 n}} \\
& =\frac{1}{2}+\sum_{n=1}^{\infty} \frac{\left(-q^{2} ; q^{2}\right)_{n-1}}{\left(q^{2} ; q^{2}\right)_{n}}(-q)^{n} \\
& =\frac{1}{2} \frac{\left(q ; q^{2}\right)_{\infty}}{\left(-q ; q^{2}\right)_{\infty}}
\end{aligned}
$$

where in the last step we used the $q$-binomial theorem $\sum_{n=0}^{\infty} \frac{(a ; q)_{n}}{(q ; q)_{n}} z^{n}=\frac{(a z ; q)_{\infty}}{(z ; q)_{\infty}}$, valid for $|z|<1$ and $|q|<1$.

From (2.1) and (2.2),

$$
\begin{aligned}
& \sum_{n=0}^{\infty} \frac{\left(q ; q^{2}\right)_{n}(-q)^{n}}{\left(-q ; q^{2}\right)_{n}\left(1+q^{2 n}\right)} p_{2 n}\left(-1 ; q^{-2 n-1},-1: q\right)-A(q) \\
& =\sum_{n=0}^{\infty} \frac{\left(q ; q^{2}\right)_{n}(-q)^{n}}{\left(-q ; q^{2}\right)_{n}\left(1+q^{2 n}\right)} \sum_{j=0}^{2 n}\left((-1)^{j}-1\right) \frac{(-1 ; q)_{j} q^{j}}{(q ; q)_{j}} \\
& =-2 \sum_{n=0}^{\infty} \frac{\left(q ; q^{2}\right)_{n}(-q)^{n}}{\left(-q ; q^{2}\right)_{n}\left(1+q^{2 n}\right)} \sum_{j=1}^{n} \frac{(-1 ; q)_{2 j-1} q^{2 j-1}}{(q ; q)_{2 j-1}} \\
& =\frac{4 q^{2}}{(1+q)} \sum_{n=0}^{\infty} \frac{\left(q^{3} ; q^{2}\right)_{n}(-q)^{n}}{\left(-q^{3} ; q^{2}\right)_{n}\left(1+q^{2 n+2}\right)} \sum_{j=0}^{n} \frac{(-q ; q)_{2 j} q^{2 j}}{\left(q^{2} ; q\right)_{2 j}} .
\end{aligned}
$$

Invoking (2.5), we see that the proof of Theorem 1.3 is complete.

\section{REFERENCES}

[1] K. Alladi, Variants of classical q-hypergeometric identities and partition implications, Ramanujan J. 31 Issues 1-2 (2013), 213-238.

[2] G. E. Andrews and R. A. Askey, Enumeration of partitions: The role of Eulerian series and q-orthogonal polynomials, Higher Combinatorics, M. Aigner, ed., Reidell Publ. Co., Dordrecht, Holland, pp. 3-26 (1977).

[3] G. E. Andrews, A. Dixit, D. Schultz and A. J. Yee, Overpartitions related to the mock theta function $\omega(q)$, Acta Arith. 181 No. 3 (2017), 253-286.

[4] K. Bringmann, C. Jennings-Shaffer and K. Mahlburg, On a modularity conjecture of Andrews, Dixit, Schultz and Yee for a variation of Ramanujan's $\omega(q)$, Adv. Math. 325 (2018), 505-532.

Department of Mathematics, Indian Institute of Technology Gandhinagar, Palaj, GandhiNAGAR, Gujarat 382355, India

E-mail address: adixit@iitgn.ac.in 